\title{
Health-related Quality of Life After Fractures of the Lateral Third of the Clavicle in Children and Adolescents
}

\author{
Thoralf R. Liebs, MD, PhD, Basil Ryser, MD, Nadine Kaiser, MD, Theddy Slongo, MD, \\ Steffen Berger, MD, PhD, and Kai Ziebarth, MD, PhD; \\ for The Bernese Pediatric Fracture Project (BPFP)
}

\begin{abstract}
Introduction: The health-related quality of life after pediatric fractures of the lateral third of the clavicle is unknown. Given the trend in the literature toward surgical management of clavicular fractures in adults, we frequently receive referrals for surgical management of lateral clavicular fractures in children. Therefore, we examined (1) the health-related quality of life of patients who sustained a fracture of the lateral third of the clavicle in childhood and who were treated at a large regional pediatric trauma center, (2) if the health-related quality of life was associated with the age at the time of the injury, and (3) whether fracture and displacement patterns were associated with health-related qualify of life.

Methods: This is a retrospective case series. We were able to identify 69 patients ( 21 girls and 48 boys) who sustained a fracture of the lateral third of the clavicle between the period spanning from January 2004 to June 2015. These patients were asked to fill in a questionnaire that included the Quick version of the Disability of Arm, Shoulder, and Hand (DASH) (primary outcome) and the Pediatric Quality of Life Inventory (Peds-QL). Until July 2017, we were able to get a response from 56 of these patients, resulting in a follow-up of $81 \%$.
\end{abstract}

From the Department of Pediatric Orthopaedics and Traumatology, Clinic for Pediatric Surgery, Inselspital Bern University Hospital, University of Bern, Bern, Switzerland.

Our institution has received research support funding from the "Batzebär" foundation, Inselspital Bern, Switzerland. "Batzebär" is an organization aimed to collect donations to support research at our hospital. Peer review is used in the process of deciding which projects are to be supported.

Each author certifies that he or any member of his or her immediate family has no funding or commercial associations (eg, consultancies, stock ownership, equity interest, patent/licensing arrangements, etc.) with the National Institutes of Health (NIH), the Wellcome Trust, Howard Hughes Medical Institute (HHMI), or other institutions that might be considered a conflict of interest.

The authors declare no conflicts of interest.

Reprints: Thoralf R. Liebs, MD, PhD, Department of Pediatric Orthopaedics and Traumatology, Clinic for Pediatric Surgery, Inselspital Bern University Hospital, University of Bern, Freiburgstrasse 4, 3010 Bern, Switzerland. E-mail: liebs@liebs.eu.

Supplemental Digital Content is available for this article. Direct URL citations appear in the printed text and are provided in the HTML and PDF versions of this article on the journal's website, www. pedorthopaedics.com.

Copyright (C) 2019 Wolters Kluwer Health, Inc. All rights reserved.

DOI: $10.1097 / \mathrm{BPO} .0000000000001332$
Results: Treatment was conservative in all cases but one. In these cases, after a mean follow-up of 6.5 years (range, 1 to $13.2 \mathrm{y}$ ), the mean Quick-DASH was 1.2 (SD, 4.4) at a scale of 0 to 100 , with lower values representing better quality of life. The mean physical function score of the Peds-QL was 97.8 (SD, 4.4), and the mean psychosocial function score was 91.8 (SD, 10.8), both at a scale of 0 to 100 , with higher values representing better quality of life. There was no statistically significant association of age at the time of injury on the primary outcome Quick-DASH. There were no statistically significant associations between fracture or displacement patterns with regard to health-related quality of life.

Conclusions: Conservative treatment of lateral clavicular fractures in children and adolescents is associated with excellent healthrelated quality of life, as measured with the disease-specific QuickDASH and the non-disease-specific Peds-QL at a mean follow-up of 6.5 years (range, 1 to $13.2 \mathrm{y}$ ). There was no effect of age at the time of injury, and we were unable to identify associations to commonly analyzed risk factors, such as displacement patterns. As the conservative treatment of this type of fracture in children and adolescents is associated with such a good health-related quality of life, it is unlikely that these results could be further improved by surgical interventions.

Level of evidence: Level IV_case series.

Key Words: fracture, clavicle, management, conservative therapy, surgery, outcome, health-related quality of life, DASH, Peds-QL

(J Pediatr Orthop 2019;39:e542-e547)

njuries of the musculoskeletal system are a remarkable common entity in children. Is has been estimated that, for children, the probability to obtain a fracture, until they have finished growth, ranges between $15 \%$ and $45 \%$, with fractures of the clavicle being especially common, with a reported frequency of $10 \%$ of all extremity fractures. Although the majority of fractures of the clavicle occur in the middle third, up to $30 \%$ of clavicular fractures occur further laterally. ${ }^{1}$

There is an ongoing controversy as to which of these fractures need surgery. ${ }^{2-4}$ As there is hardly any literature with regard to these fractures in children, some surgeons base their treatment recommendations on the experiences gained in adults, ${ }^{5}$ wherein the literature traditionally suggests surgery for lateral fractures of the clavicle, ${ }^{6,7}$ especially if the fracture is considered unstable because of a loss of stabilization by the acromioclavicular (AC) and coracoclavicular 
ligaments, ${ }^{7-12}$ owing to a high risk of nonunion or nonesthetic appearance because of callus formation. ${ }^{7}$

This could explain why there is a trend in the literature to lower the age thresholds for considering surgery for these fractures. ${ }^{13-15}$ One recent study recommended that children older than 8 years with displaced fractures of the lateral third could be treated surgically to have better cosmetic results, ${ }^{16}$ and another publication described a new surgical technique for use in children. ${ }^{4}$

Moreover, lateral clavicular fractures in children can mimic AC dislocations, ${ }^{17,18}$ an injury that is commonly seen in adults, and wherein some authors also recommend surgery. $2,7,19$

All these publications are probably the reason why we frequently see children and adolescents with lateral clavicular fractures, who are referred to us for surgical management. However, in our experience, conservative management of these fractures leads to good results. However, as there is hardly any literature on this topic, we were unable to provide evidence for our impression in the discussions with both the referring doctors and the parents.

Therefore, this study was initiated to examine (1) the health-related quality of life of patients who sustained a fracture of the lateral third of the clavicle in childhood and who were treated at a large regional pediatric trauma center, (2) whether health-related quality of life was associated with the age at the time of the injury, and (3) if health-related quality of life was associated with radiologic fracture patterns.

\section{METHODS}

This is a retrospective analysis, in which patients who underwent treatment for a fracture of the lateral third of the clavicle were contacted by postal mail. The study protocol was approved by both the Institutional Review Board of the Paediatric Clinics of Inselspital, University of Bern, and the Ethics Commission of the Canton of Bern (Basec-Nr: 2016-00011), both in Switzerland.

All sequential patients with an age of up to 16 years, who sustained a fracture of the clavicle during the period spanning from January 2004 to June 2015 and who were treated at our institution were candidates for inclusion in the study. Our institution is one of the leading peadiatric trauma centers in Switzerland, serving $>1$ million inhabitants in the German-speaking part of Switzerland.

Patients were identified on the basis of the radiologic reports within our Picture Archiving and Communication System (PACS).

For the purpose of this analysis, the inclusion criteria were limited to patients who have sustained a lateral fracture of the clavicle, wherein lateral fracture was defined as the radiographic location of the fracture within the distal third ${ }^{20}$ in relation to the full length of the clavicle. Exclusion criteria were (1) perinatal injuries or malformations, (2) other significant trauma requiring treatment, (3) inability to complete the questionnaires because of cognitive or language difficulties (STROBE participant flowchart in the electronic appendix, Supplemental Digital Content 1, http://links.lww.com/BPO/ A205).
Radiologic analysis was performed to measure the projected length of the clavicle, the location of the fracture, degree of displacement, and the amount of shortening. On the basis of these data, the location of the fracture in percentiles of the length of the clavicle was calculated. We also classified the fractures according to the system proposed by Nenopoulos et $\mathrm{al}_{1}{ }^{16}$ for which a graphical representation is available. ${ }^{21}$ The persons performing the image analysis were not aware of the patient's clinical result, thereby avoiding observer bias.

We noticed that there were several fractures that could not be classified using the Nenopoulos classification system. These were transverse fractures in the medial aspect of the lateral third, which occurred almost exclusively in the youngest age group. As these fractures were very close to the medial third, we defined them "as midshaft" and analyzed them in a separate category.

Beginning in August 2015, we have sent information about the study, a consent form, and questionnaires to the patients (or their parents, depending on calculated current age) by postal mail (STROBE participant flowchart in the electronic appendix, Supplemental Digital Content 1, http://links.lww.com/BPO/A205). Nonresponding participants were reminded 3 times by mail. Participants still not responding were contacted by phone to determine the reason for not responding. At that time, it was attempted to administer the questionnaire by phone. If participants could still not be contacted, the last known pediatrician or general physician was contacted.

We used the disease-specific Quick-DASH (Disabilities of the Arm, Shoulder, and Hand) as the primary outcome measure, for which a validated translation to our language exists. $^{22}$ Responses were recorded on a 5-point Likert scale (eg, no, mild, moderate, severe difficulty, unable). Scores were standardized to a score of 0 to 100 , with higher scores indicating more disability. If $>10 \%$ of the items were not answered, a Quick-DASH score was not calculated and regarded as missing. The minimal clinically important difference for the Quick-DASH questionnaire has been calculated to be 8 points. ${ }^{23}$

As a secondary outcome for the analysis of self-reported health-related quality of life, we have selected the Pediatric Quality of Life Inventory (Peds-QL), ${ }^{24}$ which is available in a validated German version. Scores were standardized to a score of 0 to 100 , with higher scores indicating more physical function and more social function.

Additional secondary outcomes included a question about patient satisfaction (How satisfied are you with your injured shoulder?) with very satisfied, somewhat satisfied, somewhat dissatisfied, and very dissatisfied as the possible responses, ${ }^{25}$ and a question about cosmetic appearance (How satisfied are you with the cosmetic appearance of your injured shoulder?) with not at all satisfied, a little satisfied, moderately satisfied, quite satisfied, and very satisfied as the possible responses.

Data on demographics, dates of the injury, the side (right/left), the treatment chosen, and possible revisions were collected from both the radiologic analysis and from the electronic patient chart. In the questionnaire, we included an item about handedness. 

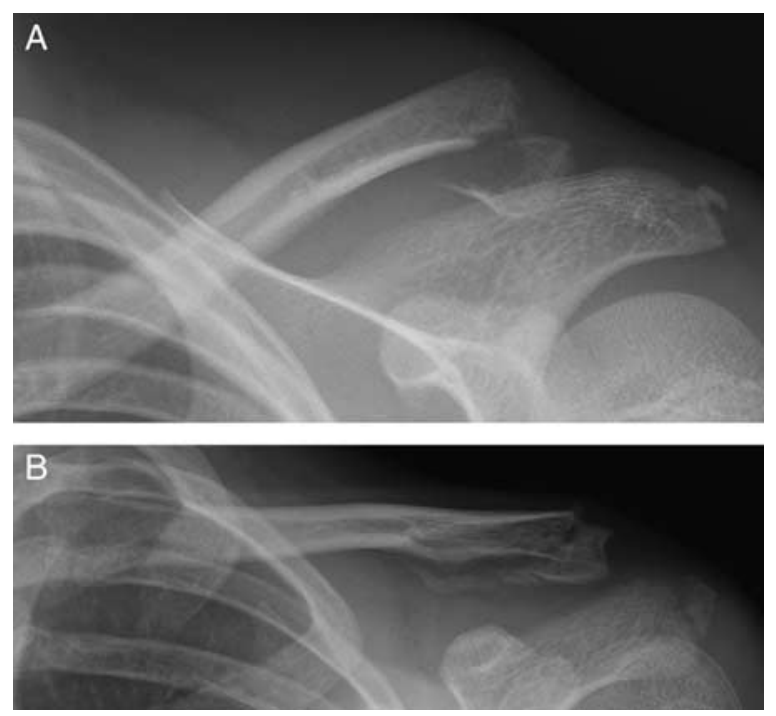

FIGURE 1. Typical radiographs at initial presentation $(A)$ and 4 weeks after nonoperative treatment (B), showing callus formation alongside the inferior border of the lateral end of the clavicle where the injury separated the periosteum from the clavicle.

Overall, we were able to identify 69 patients (21 girls, 48 boys) who sustained a fracture in the lateral third of the clavicle at an average age of 6.8 (SD, 5.1) years.

Of these, all but one were treated nonoperatively with an arm sling. We recommended that the children should wear a T-shirt while sleeping to avoid uncontrolled movements. Patients were invited to a routine follow-up after 5 days, and after 4 to 6 weeks, depending on age. A typical radiograph obtained at presentation is shown in Figure 1A, whereas a typical radiographs after 4 weeks is shown in Figure 1B.

Of the 69 cases with a lateral fracture of the clavicle, we performed surgery in one case. That was a 15 -year-old boy who fell on his shoulder while snowboarding. He sustained a type IIIb fracture according to Nenopoulos et al, ${ }^{16}$ but radiologically there was a displacement of almost 3 shaft widths. Intraoperatively, the distal end of the proximal fragment was entrapped in the trapezius muscle, from which it was mobilized and sutured to the distal fragment using drill holes. The periosteal sleeve was closed. We performed separate analysis for the patients who were treated conservatively, and for the one patient who underwent surgery.

Continuous data (Quick-DASH, Peds-QL scores) were tested initially for normal distribution with the KolmogorovSmirnov test with Lilliefors significance correction. These data were not normally distributed. As this is a case series, we have refrained from performing a power calculation.

After the description of the main outcome measure, we have performed bivariate analysis in which we have analyzed the health-related quality of life in relation to various demographic and radiologic parameters. We classified age at the time of the injury into 3 groups: $<6$, 6 to $<12$, and 12 years and older. We used the KruskalWallis and the Jonckheere Terpstra tests for comparisons.
All $P$-values are 2-tailed; no corrections were made for multiple comparisons. Statistical analysis was performed using SPSS (SPSS Inc., Chicago, IL) and R. ${ }^{26}$

\section{RESULTS}

At a mean follow-up of 6.5 (SD, 3.6; range, 1.0 to 13.2) years, we were able to follow-up on 56 patients $(81 \%)$ (Table 1$)$.

In all the 55 patients treated conservatively, the mean score for the Quick-DASH was 1.2 (SD, 4.4), the mean score for the Peds-QL-PF was 97.8 (SD, 4.4), and for the Peds-QLPS it was 91.8 (SD, 10.8), Table 2. In total, 54 patients $(98 \%)$ were overall "very satisfied" with their shoulder, and, again, 54 were "very satisfied" or "quite satisfied" with the cosmetic appearance; the one remaining patient was "somewhat satisfied" with the shoulder overall and "moderately satisfied" with the cosmetic appearance (Table 2).

There was no effect of age at the time of injury on the primary outcome Quick-DASH $(P=0.196)$ (Table 2$)$. There was a statistically significant effect of age at the time of injury on one of the secondary outcomes, the Peds-QL-PF $(P=0.009)$, with older children reporting worse function (Table 2), but the group differences did not reach the minimal clinical important difference of 8 Quick-DASH points. There was no statistically significant effect of age at the time of injury on the Peds-QL-PS $(P=0.633)$ (Table 2).

There was no statistically significant association between the primary outcome Quick-DASH and the Nenopoulos fracture classification $(P=0.581)$, displacement $(P=0.443)$, or shortening $(P=0.417)$. Furthermore, there also was no significant association of health-related quality of life, as measured by the physical function or the psychosocial functional scale of the Peds-QL, patient overall satisfaction, or satisfaction with cosmetic appearance, in relation to the fracture patterns.

One patient was treated surgically. At follow-up, he scored 6.82 on the Quick-DAHS, 93.75 on the Peds-QLPF, and 95 on the Peds-QL-PS. He was "very satisfied" overall with his shoulder, and he was "quite satisfied" with the cosmetic appearance of the shoulder. Apart from that case, there were no other surgical interventions.

\section{DISCUSSION}

This study showed that conservative treatment of fractures of the lateral third of the clavicle in children and adolescents is associated with excellent health-related quality of life, as measured with the Quick-DASH and the Peds-QL, at a mean of 6.4 years' follow-up. These excellent results were independent of commonly analyzed risk factors, such as age and radiologic fracture patterns.

These results are important, as there is a trend in the literature suggesting surgery for these fractures. For instance, one author has mentioned an age threshold for 8 years for specific fractures, ${ }^{16}$ and another author has described a new surgical technique for these fractures in children. ${ }^{4}$ At our institution, we have also noted an increasing number of referrals for surgery from external hospitals, orthopaedic surgeons, or family doctors.

Our analysis is one of the largest covering lateral fractures of the clavicle and is one of the very few assessing the 
TABLE 1. Baseline Characteristics and Radiologic Parameters

\begin{tabular}{|c|c|c|c|c|c|c|c|c|}
\hline & \multicolumn{6}{|c|}{ Age at the Time of Injury (y) } & & \\
\hline & \multicolumn{2}{|c|}{$0-<6$} & \multicolumn{2}{|c|}{$6-<12$} & \multicolumn{2}{|c|}{12 and Older } & \multicolumn{2}{|c|}{ Total } \\
\hline & $\begin{array}{c}\text { Count } \\
(\text { Col. \%) } \\
\end{array}$ & $\begin{array}{c}\text { Mean } \\
(\mathrm{SD})\end{array}$ & $\begin{array}{c}\text { Count } \\
(\text { Col. \%) }\end{array}$ & $\begin{array}{c}\text { Mean } \\
(\mathrm{SD})\end{array}$ & $\begin{array}{c}\begin{array}{c}\text { Count } \\
(\text { Col. \%) }\end{array} \\
\end{array}$ & $\begin{array}{c}\text { Mean } \\
(\mathrm{SD})\end{array}$ & $\begin{array}{c}\text { Count } \\
(\text { Col. \%) }\end{array}$ & $\begin{array}{c}\text { Mean } \\
(\text { SD) }\end{array}$ \\
\hline \multicolumn{9}{|l|}{ Sex } \\
\hline Female & $14(48)$ & - & $4(24)$ & - & $1(11)$ & - & $19(35)$ & - \\
\hline Male & $15(52)$ & - & $13(76)$ & - & $8(89)$ & - & $36(65)$ & - \\
\hline \multicolumn{9}{|c|}{ Injured side (dominat versus nondominant) } \\
\hline Nondominant side & $13(45)$ & - & $7(41)$ & - & $5(56)$ & - & $25(45)$ & - \\
\hline Dominant side & $16(55)$ & - & $10(59)$ & - & $4(44)$ & - & $30(55)$ & - \\
\hline Dislocation (radiologic) (mm) & - & $1.6(2.0)$ & - & $4.5(3.9)$ & - & $3.8(3.3)$ & - & $2.9(3.2)$ \\
\hline \multicolumn{9}{|c|}{ Dislocation (radiologic) in shaft widths } \\
\hline None & $24(83)$ & - & $17(100)$ & - & $9(100)$ & - & $50(91)$ & - \\
\hline No shortening & $22(76)$ & - & $10(59)$ & - & $8(89)$ & - & $40(73)$ & - \\
\hline$>0 \%-5 \%$ & $2(7)$ & - & $5(29)$ & - & $1(11)$ & - & $8(15)$ & - \\
\hline$>5 \%-10 \%$ & $4(14)$ & - & - & - & - & - & $4(7)$ & - \\
\hline$>10 \%-15 \%$ & $1(3)$ & - & $1(6)$ & - & - & - & $2(4)$ & - \\
\hline$>15 \%$ & - & - & $1(6)$ & - & - & - & $1(2)$ & - \\
\hline \multicolumn{9}{|c|}{ Radiologic fracture classification* } \\
\hline I & $6(21)$ & - & $3(18)$ & - & $2(22)$ & - & $11(20)$ & - \\
\hline IIa & - & - & $2(12)$ & - & - & - & $2(4)$ & - \\
\hline $\mathrm{IIb}$ & $1(3)$ & - & $2(12)$ & - & $2(22)$ & - & $5(9)$ & - \\
\hline IIIa & $2(7)$ & - & $2(12)$ & - & $3(33)$ & - & $7(13)$ & - \\
\hline $\mathrm{IIIb}$ & $2(7)$ & - & $7(41)$ & - & $2(22)$ & - & $11(20)$ & - \\
\hline IV & - & - & - & - & - & - & - & - \\
\hline
\end{tabular}

Col. \% indicates column percentage.

*Modified radiologic fracture classification according to Nenopoulos and colleagues, with the added category "as midshaft." Please refer to the Methods section for description.

health-related quality of life after these typical accidents in this patient population. However, there are limitations: First, as this was a monocenter study, it could be argued that external validity is limited. However, as we are the only institution in a wide geographical area covering pediatric trauma, and we included all sequential patients suffering from this injury, a bias in the run-in phase is unlikely, making a high external validity probable. Second, there is no universally accepted classification system for the radiologic assessment of these fractures, and we followed the definition that a lateral fracture of the clavicle is located in the distal third. ${ }^{20}$ We have utilized the most recent system suggested by Nenopoulos et al. ${ }^{16,21}$ Although this is considered to be a modern system, ${ }^{4}$ we were not able to sufficiently allocate every fracture within this system. This included transverse fractures located on the medial aspect of the lateral third, which appeared to represent a midshaft fracture of the clavicle, although the fracture localization was distal to the middle third. To account for these fracture patterns, we added a category to the Nenopoulos classification termed "as midshaft"; however, there was no statistically significant different health-related quality of life when these fractures were compared with the other fracture types. Given the excellent results of mainly nonoperative treatment in this patient group, it appears unlikely that a different radiologic system would have altered the results of this study. Third, the examined radiographs were made routinely at presentation to our institution, and were not specifically prepared for this analysis. Therefore, the setting of these radiographs is comparable to the situation of the clinician. However, it raises the question as to how accurate the measurements performed in our analysis are. As we have measured predefined distances on the radiograph and calculated ratios on a spreadsheet, we have made every effort to limit measurement errors. In addition, the person identifying the relevant structures on the image was not aware of the clinical result of the patient; thereby, the radiologic assessment could be regarded as blinded. Fourth, as this study has a retrospective design, our study suffers from methodological weaknesses common in this design. This includes missing data on the health-related quality of life before the injury. Although this is considered a 
TABLE 2. Health-related Quality of Life and Patient Satisfaction After Fracture of the Lateral Clavicle by Age at the Time of Injury

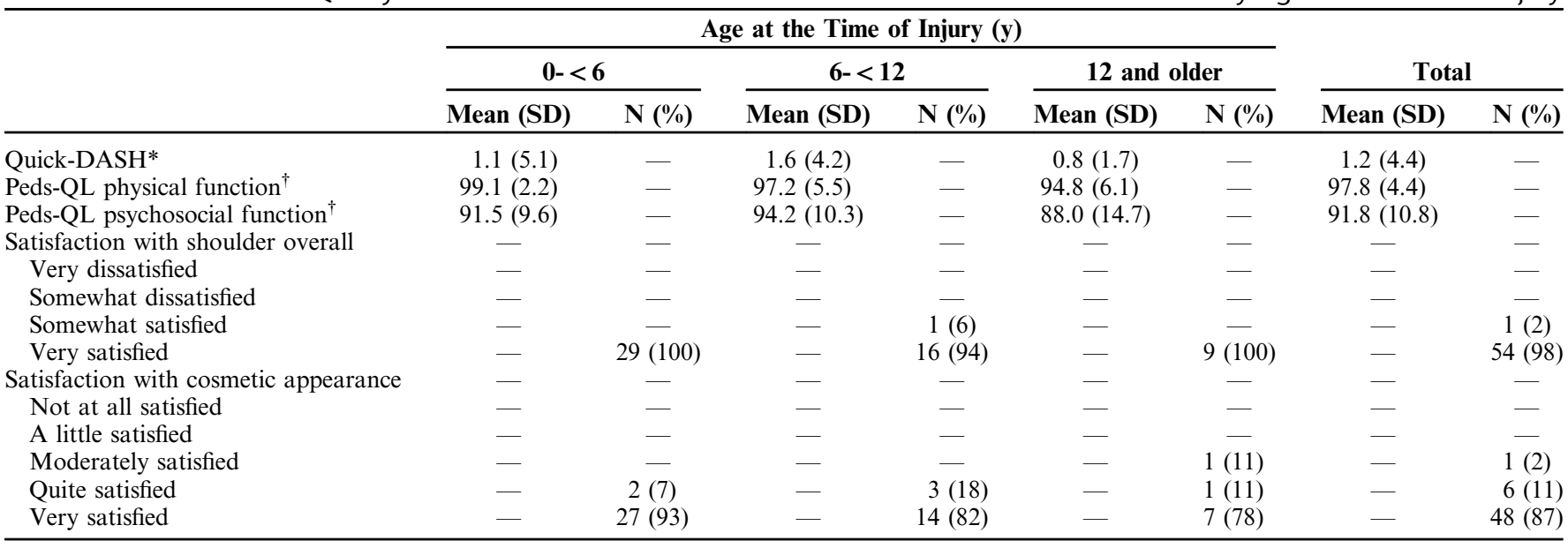

* Range from 0 to 100 , with lower values representing better quality of life.

$\dagger$ Range from 0 to 100 , with higher values representing better quality of life.

DASH indicates Disability of Arm, Shoulder, and Hand; Peds-QL, Pediatric Quality of Life Inventory.

methodological weakness in studies analyzing adult fractures, this does not apply to pediatric fractures, as children usually have no physical limitations before an injury. Therefore, the assumption that limitations of the diseasespecific outcome measure used in this study are in fact attributable to the injury should be allowed. Fifth, the QuickDASH has not yet been formally validated in this age group, and, in some cases, the parents filled in the questionnaire on the basis of their perceptions of their children's functioning, which might explain a ceiling effect of the instrument. However, the Quick-DASH has been used by several authors for evaluation of children with elbow fractures, clavicular fractures, or other upper extremity fractures. Therefore, the DASH/Quick-DASH seems to be the most commonly used outcome measure for pediatric fractures of the upper extremities. Sixth, owing to the sample size, it is possible that we missed identifying existing associations of health-related quality of life with age or fracture patterns. Given the excellent results of the overall group, it is unlikely that any such association would gain clinical relevance, if they existed. Seventh, as sports that require a throwing or overhead activity with the dominant arm do no play a major role in our country, we are unable to report on this specific aspect of health-related quality of life that might have greater importance in other countries. Unfortunately, the QuickDASH does not include a question that addresses this issue; therefore, we are unable to comment on this aspect. Eighth, our analysis was restricted to patients up to 16 years at the age of the injury. Therefore, these results should not be extrapolated to patients older than this age.

The main focus of our study was to report the healthrelated quality of life of our population. In our cohort of 56 predominantly conservatively treated children and adolescents who answered our questionnaire, there were exceptionally good mean scores for the primary outcome Quick-DASH and the secondary outcomes Peds-QL-PF and Peds-QL-PS. This finding contradicts the current trend to perform surgery in this injury, which traditionally is treated nonoperatively. It is the policy of our institution to limit the indications for surgery to open fractures or fractures with a risk of skin perforation, neurovascular injuries, or entrapment of soft tissue or muscles preventing bone healing.

In an earlier publication from our institution, it was concluded that the results are satisfactory in children with any kind of clavicular fracture without major complications when surgically treated; however, that analysis was limited to patients who underwent surgery, ${ }^{27}$ lacking a nonoperative comparison group. In a more recent publication, it has been reported that of 75 patients with a lateral clavicular fracture, 12 received surgery, and 52 patients were able to follow instructions for the assessment of the Constant score, and all of these had the maximum score $\left(100\right.$ of 100). ${ }^{16}$ Although it seems statistically unusual that a maximum score of 100 could be attained in all 52 patients who attended the follow-up, the message of the authors remains that there are good clinical results in children with lateral fractures of the clavicle. However, it is unknown whether the results of that study were obtained because the authors performed surgery, or in spite of performing surgery. Although there are some other studies reporting clinical results, none of those used

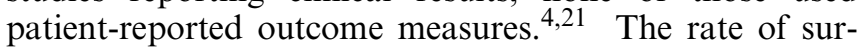
gery with 1 case in 69 patients was low in our cohort. Other authors have reported comparable low rates of surgery in such patient groups. ${ }^{21,27}$

As other authors appear to have lowered the age threshold for performing surgery in children, we analyzed the effect of age at the time of injury. We could not demonstrate an association of the effect of age at the time of the injury in relation to the main outcome measure. As the results of our predominantly conservatively treated cohort were excellent in all age groups, our results do not justify performing 
surgery in the age groups analyzed here. For one of the secondary outcomes, there was a statistically significant effect of age, with patients in the older age groups demonstrating less quality of life, as measured with the PedsQL-PF, than in the younger age groups. However, the absolute group differences were small and did not reach the threshold for a minimal clinically important difference. ${ }^{23}$

We could not identify risk factors in terms of radiologic fracture classification, displacement, or shortening with regard to health-related quality of life or patient satisfaction. The Nenopoulos fracture classification system that was used in this study did not prove to be clinically relevant, as there were no associations between fracture pattern and clinical outcome, even after we added a category to that system. We also found no cases of true AC dislocations, which is considered an item of the Nenopoulos classification ${ }^{16}$; in this respect, our results are compatible with other authors who also have reported that true $\mathrm{AC}$ dislocations are rare in the pediatric population. ${ }^{3,17}$ In addition, we also found no effect of radiologically measured displacement on the health-related quality of life, thereby supporting the impression that pseudodislocations do not require surgical interventions, ${ }^{17}$ an impression that has been questioned in the past. ${ }^{2,7,19}$ As regards clavicular shortening, our study is not in accordance with the study of Randsborg et $\mathrm{al}^{28}$ who reported an association of shortening with health-related quality of life. ${ }^{28}$ This author did not define a threshold for shortening and reported that the effect of shortening was small and that their study was based mainly of midshaft fractures, with only 13 lateral fractures in the cohort. ${ }^{28}$ However, our finding is in accordance with the study authored by Nordqvist et al, ${ }^{29}$ who also was unable to demonstrate a clinical relevance of shortening. Considering these findings, it could be discussed to limit the radiologic interpretation to the question of whether there is a fracture at all and whether there is such a large amount of displacement that makes an entrapment of the proximal fragment in the muscle likely. Only in such a case did we perform surgery, which consisted of open reduction and closing of the ruptured periosteum, without hardware such as K-wires, screws, or intramedullary devices. ${ }^{30}$

In conclusion, we report excellent health-related quality of life in children and adolescents who sustained a fracture of the lateral third of the clavicle and who were mainly treated nonoperatively, making it unlikely that these excellent results could be further improved by surgery. This information is essential when discussing indications for surgery with parents and referring doctors.

\section{REFERENCES}

1. Stanley D, Trowbridge EA, Norris SH. The mechanism of clavicular fracture. A clinical and biomechanical analysis. J Bone Joint Surg Br. 1988:70:461-464.

2. Falstie-Jensen S, Mikkelsen P. Pseudodislocation of the acromioclavicular joint. J Bone Joint Surg Br. 1982;64:368-369.

3. Eidman DK, Siff SJ, Tullos HS. Acromioclavicular lesions in children. Am J Sports Med. 1981;9:150-154.

4. Schilcher J, Scheer J, Adolfsson L. Transclavicular osseous sutures for the treatment of displaced distal clavicular fractures in children. $J$ Orthop Trauma. 2016;30:e181-e185.
5. Carry PM, Koonce R, Pan Z, et al. A survey of physician opinion: adolescent midshaft clavicle fracture treatment preferences among POSNA members. J Pediatr Orthop. 2011;31:44 49.

6. Zenni EJ Jr, Krieg JK, Rosen MJ. Open reduction and internal fixation of clavicular fractures. J Bone Joint Surg Am. 1981;63:147-151.

7. Seppel G, Lenich A, Imhoff AB. Distal clavicle fracture. Oper Orthop Traumatol. 2014;26:254-262.

8. Edwards DJ, Kavanagh TG, Flannery MC. Fractures of the distal clavicle: a case for fixation. Injury. 1992;23:44-46.

9. Flinkkila T, Ristiniemi J, Hyvonen P, et al. Surgical treatment of unstable fractures of the distal clavicle: a comparative study of Kirschner wire and clavicular hook plate fixation. Acta Orthop Scand. 2002;73:50-53.

10. Kalamaras M, Cutbush K, Robinson M. A method for internal fixation of unstable distal clavicle fractures: early observations using a new technique. J Shoulder Elbow Surg. 2008;17:60-62.

11. Kashii M, Inui H, Yamamoto K. Surgical treatment of distal clavicle fractures using the clavicular hook plate. Clin Orthop Relat Res. 2006;447:158-164.

12. Robinson CM, Akhtar MA, Jenkins PJ, et al. Open reduction and endobutton fixation of displaced fractures of the lateral end of the clavicle in younger patients. $J$ Bone Joint Surg Br. 2010;92:811-816.

13. Li Y, Helvie P, Farley FA, et al. Complications after plate fixation of displaced pediatric midshaft clavicle fractures. J Pediatr Orthop. 2018;38:350-353

14. Vander Have KL, Perdue AM, Caird MS, et al. Operative versus nonoperative treatment of midshaft clavicle fractures in adolescents. J Pediatr Orthop. 2010;30:307-312.

15. Namdari S, Ganley TJ, Baldwin K, et al. Fixation of displaced midshaft clavicle fractures in skeletally immature patients. $J$ Pediatr Orthop. 2011;31:507-511.

16. Nenopoulos SP, Gigis IP, Chytas AA, et al. Outcome of distal clavicular fracture separations and dislocations in immature skeleton. Injury. 2011;42:376-380.

17. Black GB, McPherson JA, Reed MH. Traumatic pseudodislocation of the acromioclavicular joint in children. A fifteen year review. Am J Sports Med. 1991;19:644-646.

18. Ogden JA. Distal clavicular physeal injury. Clin Orthop Relat Res. 1984;188:68-73.

19. Dartoy C, Fenoll B, Hra B, et al. Epiphyseal fracture-avulsion of the distal extremity of the clavicle. Apropos of a case. Ann Radiol (Paris). 1993;36:125-128.

20. Allman FL Jr. Fractures and ligamentous injuries of the clavicle and its articulation. J Bone Joint Surg Am. 1967;49:774-784.

21. Labronici PJ, da SR Jr, Franco MV, et al. Distal clavicle fractures in children. Rev Bras Ortop. 2016;51:24-28.

22. Germann G, Harth A, Wind G, et al. Standardisation and validation of the German version 2.0 of the Disability of Arm, Shoulder, Hand (DASH) questionnaire. Unfallchirurg. 2003;106:13-19.

23. Mintken PE, Glynn P, Cleland JA. Psychometric properties of the shortened disabilities of the Arm, Shoulder, and Hand Questionnaire (QuickDASH) and Numeric Pain Rating Scale in patients with shoulder pain. J Shoulder Elbow Surg. 2009;18:920-926.

24. Mahan ST, Kalish LA, Connell PL, et al. PedsQL correlates to PODCI in pediatric orthopaedic outpatient clinic. J Pediatr Orthop. 2014;34:e22-e26.

25. Liebs TR, Herzberg W, Ruther W, et al. Ergometer cycling after hip or knee replacement surgery: a randomized controlled trial. $J$ Bone Joint Surg Am. 2010;92:814-822.

26. R Development Core Team. R: A Language and Environment for Statistical Computing. Vienna, Austria: R Foundation for Statistical Computing; 2008.

27. Kubiak R, Slongo T. Operative treatment of clavicle fractures in children: a review of 21 years. J Pediatr Orthop. 2002;22:736-739.

28. Randsborg PH, Fuglesang HF, Rotterud JH, et al. Long-term patient-reported outcome after fractures of the clavicle in patients aged 10 to 18 years. J Pediatr Orthop. 2014;34:393-399.

29. Nordqvist A, Redlund-Johnell I, von Scheele A, et al. Shortening of clavicle after fracture. Incidence and clinical significance, a 5-year follow-up of 85 patients. Acta Orthop Scand. 1997;68:349-351.

30. Itokazu M, Yoshida M, Itoh Y, et al. Trapezius interposition of a distal third clavicular fracture in a child: a case report. J Orthop Surg (Hong Kong). 2001;9:67-69. 